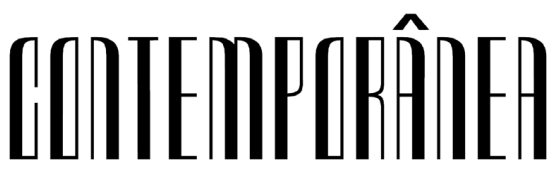

ISSN (Eletrônico): 2316-1329

http://dx.doi.org/10.4322/2316-1329.009

Contemporânea

v. 6, n. 1, p. $75-95$

Jan.-Jun. 2016

Dossiê

\section{Gubernamentalidades futuristas: gobernar el hogar en la era del optimismo tecnológico}

Pablo Jaramillo ${ }^{1}$

Resumen: Este artículo analiza la obsesión con las tecnologías de la información y otras formas de mediación técnica en la asistencia social en Colombia en los últimos 10 años, tres programas sociales distintos (en La Guajira y Bogotá). Su conclusión principal es que la relación entre tecnología y asistencia tiene dos tipos de efectos complementarios: por un lado crea una política social incorporada en mediadores materiales que modifican la relación burocrática. Por el otro, apunta a gobernar la intimidad del 'hogar' marginal (en términos étnicos, raciales, socioeconómicos y de nivel educativo) sin la intrusión de funcionarios y así modelarlo como una unidad de intervención y, a sus integrantes, agentes funcionales de la ejecución de la política social.

Palabras claves: programas de transferencia condicionas en efectivo; tecnología; asistencia social; marginalidad; moneda; innovación social.

Futuristic governmentality: governing the home in the age of technological optimism

Abstract: This article delves into the obsession for information technologies and other forms of technical mediation in social assistances in Colombia during the last decade, in three different social programs. The main conclusion is that the relationship between technology and assistance is twofold: on the one hand, it creates a social policy embodied in material stuff making easy to replace bureaucratic models of

${ }^{1}$ Universidad de los Andes, Bogotá - Colômbia - e-mail: p.jaramillo23@uniandes.edu.co 
policy. On the other, it aims to govern the intimacy of marginal (in ethnic, racial socioeconomic and education terms) 'homes' through devices that have an in-built model of relationships apt for the kind of hegemonic development envisaged for the country.

Keywords: conditional cash transfer programs; technology; social assistance; marginality; money; social innovation.

\section{Introducción}

Rosa Bogotá, un mujer pobre, beneficiaria y líder del programa Familias en Acción, revisa su correo electrónico y alimenta una hoja de cálculo en Excel a través de la cual ha logrado manejar una amplia su propio trabajo con una empresa de correos. También, cada día de por medio, lee la prensa y revisa sus indicadores de seguridad social que la hacen beneficiaria de programas de asistencia y, a veces los de las mujeres vecinas. Cada día revisa su Facebook donde sus amigos y amigas postean sus selfies y, de vez en cuando circulan noticias sobre los programas sociales y memes que condenan la dependencia que crean. Dolores Pushaina, una mujer indígena wayúu, escucha la radio en su ranchería: un comercial le indica que "actualice sus datos", sin lo cual no podrá reclamar su subsidio 3 . Termina de preparar el chivo que su hijo acaba de sacrificar, sirve el desayuno y se pone su manta para ir con urgencia al pueblo a hablar con un funcionario que le ayude con su actualización. Las dos mujeres no se conocen, pero sus vidas son atravesadas por la obsesión gubernamental de gobernar el hogar pobre a través de tecnologías de la información. El objeto de este artículo es caracterizar la suerte de optimismo tecnológico (Basiago, 1994) de la asistencia social a las "poblaciones vulnerables" en Colombia (una categoría usada por la política social, más no por los sujetos de las mismas, quienes se refieren a sí mismos como pobres, indígenas u otras categorías étnicas y raciales) contemporánea como una forma de gubernamentalidad futurista del hogar y, por ende, de la intimidad. También ofrece un análisis a la vez longitudinal y etnográfico producto de una década de etnografía sobre la asistencia social en Colombia. El objeto de este artículo es, de alguna manera, cómo su tema central llego a ser aparente.

Las metáforas tecnológicas son centrales para la manera como el Estado colombiano se representa a sí mismo contemporáneamente: se supone que opera a través de "articulaciones", "encadenamientos", "engranajes", "sinergias". Estas nociones y las tecnologías que la respaldan son un desafío para los antropólogos y sociólogos del estado contemporáneo. El análisis de las prácticas de la asistencia social fue heredero de los estudios sobre la burocracia en ciencias sociales. Dicho modelo,

${ }^{3}$ El pueblo indígena wayúu vive actualmente en la frontera colombo-venezolana en la península de La Guajira. Son más o menos 500000 y habitan en su territorio tradicional y las ciudades vecinas. Son un pueblo fuertemente jerarquizado y se organizan en familias extensas matrilineales y redes de aliados. Subsisten, principalmente, del pastoreo y del comercio. 
de herencia Weberiana y mejorado por transaccionalistas como Don Handelman (1976), pone gran peso en las interacciones cara a cara entre funcionario y cliente. Esta suerte de relación entre ciudadano y estado se ha visto transformada radicalmente por la aspiración tecnocrática, basada en el gobierno del diseño de la política pública (Centeno, 1997). En este sentido, el modelo burocrático tiene un alcance limitado para comprender las formas emergentes de asistencia. Es cierto que el contacto directo y no mediado de las relaciones burocrática más analizadas etnográficamente (cf. Herzfeld, 1993) continúa teniendo su lugar en las relaciones entre el Estado y los beneficiarios de los programas sociales. No obstante, es clave tener en cuenta que, influidos por el lenguaje de la eficiencia y la transparencia, los estados contemporáneos (y muy particularmente el colombiano) se ven a sí mismos como gobernados por el diseño de sus políticas, los conocimientos expertos y las tecnologías. En resumen, el modelo para comprender políticas sociales contemporáneas debe incorporar de una manera más coherente el tipo de relaciones típicas de la tecnocracia, su relación con las prácticas burocráticas que han permanecido inalteradas y la lógica política que estructura, de manera más general, las relaciones entre poder soberano y ciudadanos.

De manera más general, la atención sobre los agentes no humanos en las relaciones sociales ha sido un tema extendido en las ciencias sociales (Latour, 2005; Law, 2007) y, particularmente en las colombianas, como lo evidencia el inmenso proyecto "Ensamblado en Colombia" (Restrepo Forero, 2013a, 2013b). Como lo señalé en su momento (Jaramillo, 2013) el marco de análisis de la teoría del actor red o de las semiótica material, aunque fundamental para hacer aparente el problema, no agota el desafío que representan las nuevas tecnologías en el contexto de la asistencia social y el gobierno de poblaciones. Lo anterior es resultado, en primer lugar, de que su carácter político es mucho más explícito que otro tipo de tecnologías (cf. Winner, 1980; Latour, 1988). En segundo lugar, la inclusión de las tecnologías en la asistencia ocurre en arreglo a visiones del Estado más intencionadas, en este caso, hacia la difusión territorial, la eficiencia técnica y el gobierno de la asistencia en el cual los asistidos son responsables por su propia superación. En este sentido, este artículo adopta, entre líneas, un marco analítico más afín a las redes del poder político propuestas por Michael Mann (1986), en las cuales el problema consiste en qué tan intensivo o difuso es el gobierno que emana de las tecnologías para definir que clase de Estado, política social y sujeto asistido emerge de las relaciones en las cuales personas y artefactos son mutuamente constitutivos.

Lo anterior es particularmente importante para Colombia. Los programas sociales se ven como focos de innovación tecnológica y la innovación tecnológica se ha convertido en una forma de asistencia en sí misma. Se puede empezar por los artefactos más convencionales: formularios, encuestas, carpetas, portafolios, juegos (e incluso el dinero mismo entregado en algunos programas); todos implican mediaciones materiales que hacen funcionar la asistencia a través del diseño. Asimismo, las tecnologías de la información en comunicaciones (TICs) 
se han vuelto centrales para la administración de los programas sociales y en la experiencia que los beneficiarios tienen de la asistencia social. Colombia también ha adoptado el lenguaje de las TICs para el desarrollo (ICT4D, por sus siglas en inglés) y ha establecido específicamente un ministerio para la promoción de la política pública y la superación de la "brecha tecnológica" (el bajo acceso de personas marginales a las TICs). En resumen, las TICs son una forma de política social en sí misma y un elemento clave en el funcionamiento de la ya existente. Esto ha tomado el nombre de "innovación social" en las esferas gubernamentales. El optimismo en el diseño de la política social, incorporado particularmente en las tecnologías de la información, y las TICs como política social deben ser entendidas como un movimiento común.

Ahora bien, el objetivo último de esta innovación social está orientado a los "hogares". El "hogar", en este contexto, no es concebido como un objeto de intervención, sino como un agente que crea el desarrollo. Lo anterior implica estrategias de gobernar la intimidad que, bien ha sido analizado (Zelizer, 2011; Foucault, 1991), toma como punto de partida discursos sobre la sexualidad, el parentesco y la crianza. Oswin y Olund (2010) han propuesto, en este sentido, a la "intimidad" como dispositivo biopolítico. Pero casi toda la literatura en este sentido se ha concentrado en la sexualidad. Sin embargo, el gobierno de la intimidad en el caso de las políticas de la asistencia en Colombia también implica otros niveles de práctica que se vinculan con la noción de dar a los hogares las capacidades para ser agentes de desarrollo y estas, como mostraré son fundamentalmente soluciones tecnológicas o mediadas por artefactos. Esto no es ninguna coincidencia pues el desarrollo mismo se ha promocionado como un asunto de capacidades (Sen, 2001) y en el contexto tecnocrático esto divide a los hogares y sus miembros entre hábiles e inhábiles; esto es, entre personas capaces e incapaces de vincularse con el desarrollo. Si bien la tecnología se convierte en una manera de hacer realidad esta potencialidad de "elegir", el fin último de la ideología liberal (Kleine, 2013). El gobierno tecnologizado del hogar, como cualquier otro y como se verá al final de este artículo, no es unilateral ni exento de apropiaciones no previstas.

El hogar se convierte en un objeto de intervención material. Pero también un objeto metafórico, en la medida en que la nación tiene la posibilidad de reinventarse como "hogar" (Brickell, 2012). En este sentido la asistencia contemporánea en Colombia, con su obsesión tecnológica, también crea una geografía "del hogar", una geografía de la pobreza y la asistencia basadas en la habilidad e inhabilidad sobre la técnica del propio gobierno del hogar. Dichas geografías reflejan, de manera más general, las geopolíticas del conflicto armado y la marginalización neoliberal. Para las personas, sin embargo, esta dualidad entre capacidad e incapacidad es demasiado simplista y, en cambio, han adoptado esta gubernamentalidad futurista a través de formas tan diversas como el reciclaje tecnológico (Thomas, 1995), a través del cual logran el rediseño del artefacto para fines no anticipados. Pero también, el deseo por el artefacto y la tecnología ha conducido a personas al involucramiento en el robo de aparatos como celulares y computadores; esto 
es, un nuevo escenario de criminalización los pobres. En este artículo analizo tres momentos del optimismo tecnológico sobre la asistencia, tres programas sociales clave de la reciente política social en Colombia. En cada uno de estas instancias analizó la articulación específica de tecnologías y artefactos que median la asistencia y crean hogares gobernables. Estos programas también corresponden a momentos que se superponen en la transformación de la asistencia. El primer momento corresponde a la aplicación de Familias en Acción del año 2001 al 2010. Posteriormente, me concentro en la aplicación de Más Familias en Acción (la mutación del primer programa en el contexto de un nuevo programa presidencia) del año 2010 al presente. El tercero, corresponde a la Red Unidos (antes llamada Juntos), un programa social inicialmente establecido en el 2006, pero me concentro en el uso reciente de formas de zonificación de la pobreza a través de la estrategia conocida como ZOLIP (Zonas Libres de Pobreza Extrema). Cada uno de estos momentos corresponden a distintos proyectos de investigación que se han interesado incrementalmente en la asistencia social como problema empírico y teórico.

\section{Familias en Acción (2001-2010)}

Familias en Acción es un programa de transferencias condicionadas en efectivo. Transfiere entre 9,6 y 24,1 USD por concepto de nutrición y entre 7,2 y 28,9 USD por educación. Entrega a madres un subsidio monetario para cada hijo por concepto de nutrición y educación condicionado a la entrega de "compromisos": medios de certificar la asistencia a citas médicas regulares y al colegio (Fiszbein et al., 2009). En el año 2001, el programa fue planeado para que su aplicación ocurriera en municipios rurales (300 inicialmente), todos ellos con altos índices de pobreza y en los cuales se vivía con crudeza el conflicto armado $^{4}$. Esta decisión corresponde a la naturaleza política con la que nació el programa: fue, explícitamente, el componente social del Plan Colombia, el plan antisubversivo de Estados Unidos que se viene implementando desde 1999 (United States Government Accountability Office, 2008). Mientras que la ayuda europea fue más orientada hacia la financiación de casos específicos de desarrollo dirigido

\footnotetext{
${ }^{4}$ El conflicto armado en Colombia se remonta, por lo menos, hasta los años 1950, escenario de surgimiento de guerrillas izquierda (principalmente FARC-EP, ELN y, posteriormente el M19 y ejércitos ilegales de derecha vinculados con el narcotráfico y la ganadería extensiva. Las fuerzas militares y el estado han se han enfrentado frontalmente con las primeras mientras que frente a las segundos han (conocidos como paramilitares) En el centro del conflicto se ha encontrado una disputa por el territorio y, desde los años 1980 por el control de territorios productores de drogas ilícitas. Inicialmente los principales enfrentamientos se dieron entre fuerzas militares y guerrillas de izquierda. Durante los años 1980, los carteles de la droga y el estado financiaron los paramilitares con fines contrainsurgentes y desde eso se consolidó una estrecha, pero ambigua relación entre estos tres sectores. Durante el gobierno de Álvaro Uribe Vélez (2002-2006/2006-2010) se produjo una desmovilización de los grandes estructuras paramilitares con la promesa de un proceso de reparación a las víctimas. Mientras que la reparación ha avanzado, no todos los paramilitares dejaron sus armas y se han consolidado en zonas clave del tráfico de droga. Mientras tanto la respuesta a las guerrillas fue mucho más frontal en términos militares, hasta que el gobierno de Juan Manuel Santos (2010-2014/2014-presente) inició los diálogos con las FARC-EP en la Habana.
} 
a la reducción del conflicto social ("laboratorios de paz), siguiendo una tradición que inició con la Doctrina de la Seguridad Nacional, la ayuda norteamericana implementó Familias en Acción en los "territorios enemigos" (Gloria Puerta, comunicación personal). La financiación del programa fue, pues, directamente resultado de la ayuda militar norteamericana y posteriormente provino de la banca multilateral. A la focalización geográfica en áreas de conflicto armado se le sumó la focalización a través de medios (means testing) con del Sistema de Selección de Beneficiarios de Programas Sociales (SISBEN), una base de datos de hogares vulnerables basada en una encuesta de hogares. En este momento el programa contaba con cerca de 340 mil beneficiarias. La puerta de entrada al programa para las personas desplazadas, aunque siendo muchas de ellas registradas en el SISBEN, fue una base de datos paralela conocida en un inicio como Sistema de Información de Población Desplazada (SIPOD) y actualmente integrada al Registro Único de Víctimas (RUV).

En el año 2004, el programa fue expandido a la población desplazada en el país ${ }^{5}$. La inclusión de la población desplazada es diagnóstica, pues justamente es aquella que salió de los municipios más afectados por el conflicto armado, es decir, aquellos donde se estaba aplicando FA en primer lugar. La inclusión de desplazado también implicó la expansión del programa a todo el país, aunque no por igual para todos los lugares.

Para el año 2006, los beneficiarios del programa habían aumentado hasta 700 mil; en parte, esto se debió a la inclusión de los desplazados, pero también a que el número de municipios había llegado a 848. En un momento clave que coincidió con el inicio del segundo periodo presidencial de Álvaro Uribe Vélez, el programa se implementó en las grandes ciudades aumentando su población hasta 2,8 millones beneficiarios. También fue el momento en el que el programa llegó a los pueblos indígenas y, específicamente a La Guajira, una península semidesértica al norte de Colombia donde me encontraba haciendo trabajo de campo para mi tesis doctoral ${ }^{6}$. El lugar había sido fuertemente golpeado por la violencia paramilitar desde, al menos, 1998, cuando fuerzas armadas vestidas de civil intentaron tomar las rutas de la península por las cuales los indígenas wayúu habían contrabandeado toda clase de productos (Jaramillo, 2014). Por lo mismo, los indígenas habían sido un problema crónico para el estado colonial, primero, y el republicano, después. Los paramilitares fueron ayudados por las fuerzas militares en una campaña de sangrienta de pacificación. Como en otros lugares de país, la violencia fue seguida de la calma tensa que vino con "las

\footnotetext{
${ }^{5}$ en parte siguiendo las recomendaciones de la Corte Constitucional, que exhortaba al diseño de política pública para esta población a través de la Sentencia T-025.

${ }^{6}$ El trabajo de campo tuvo una duración de 13 meses con organizaciones y familias wayúu víctimas del conflicto armado en La Guajira. Justamente el problema de entrada de este trabajo de campo era la transformación de las políticas multiculturales con la entrada de políticas orientadas a paliar la vulnerabilidad. Esta entrada de fuerzas de ocupación no tuvo solamente un poder transformativo en términos de política social, sino que creo un escenario completamente nuevo para que las personas se reimaginaran como indígenas-pobres. Un análisis completo de esto puede encontrarse en Jaramillo (2014).
} 
ayudas" (como se le conoció cotidianamente a los subsidios estatales). La relación entre el programa FA y la ayuda humanitaria a las víctimas estaba reflejada en el hecho de que Acción Social, la agencia que administraba el programa de TCE, también ejecutaba la Ley de atención a las víctimas. En este momento el programa alcanzó los 2,5 millones de hogares (Agencia Presidencial para la Acción Social y la Cooperación Internacional, 2010)

La Guajira, donde vive Dolores la mujer wayúu mencionada en la introducción, fue uno de los primeros territorios indígenas donde Familias en Acción hizo presencia. Lo hizo, como me lo dijo su entonces coordinador, con el fin de "hacer presencia del estado". En este caso, en vez de usar la base de datos del SISBEN, FA usó unos formularios especialmente diseñados para que la población indígena se censara a sí misma (Figura 1). A los líderes de cada comunidad, que habitan rancherías, aldeas dispersas unidas por vínculos de parentesco (matrilineal) y cuya vecindad implica alianzas (matrimoniales, bélicas, entre otras), se les entregaron formularios para ser llenados casa por casa. También se les dio la instrucción de transcribir la información en una hoja de cálculo de Excel y entregar un CD con la información a las oficinas del programa.

Con los formularios, la relación burocrática se volvió más mediada y gobernada por el artefacto. También se trasladó de las oficinas a la vida cotidiana de la ranchería. Me encontraba viviendo con una familia indígena en ese momento y las conversaciones en la cocina y en horas de la noche (los momentos de verdadera intimidad para las familias) se volcaron a "los beneficios" que iban a llegar. El lugar también empezó a ser visitado por familiares lejanos y miembros de rancherías distantes. Yo ayudé a una líder a censar su propia ranchería y pude notar que no todas las casas de su parentela estaban siendo encuestadas y no todas las casas encuestadas eran de su parentela. Lo mismo estaba ocurriendo en otros lugares.

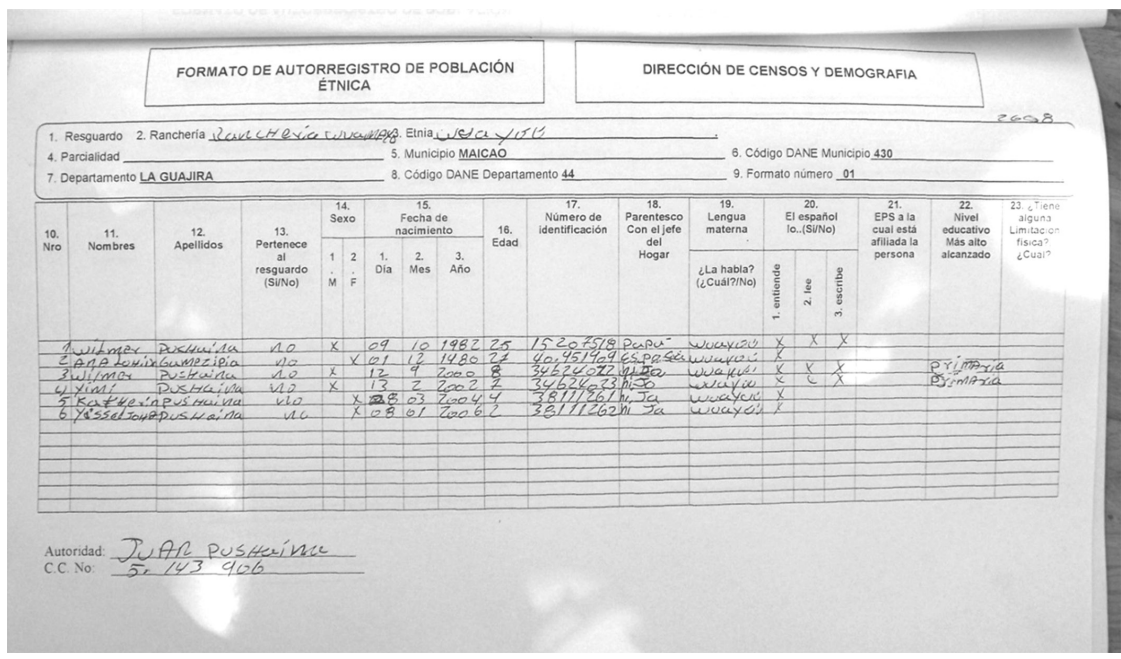

Figura 1 - Formato de Autorregistro de Población Étnica del programa FA en La Guajira Fuente: Tomada por el autor 
En ocasiones, personas que habitaban muy lejos de las rancherías terminaban incluidas en los censos de una "comunidad", como se evidencia en los dos mapas siguientes. En el primero se muestra qué "casas" censaron los líderes (Figura 2). En el segundo (Figura 3) se muestra como quedó presentada una comunidad en el censo y como un artefacto crea nuevas formas de "comunidad" modular, fragmentaria y desterritorializada. A través de la encuesta, el hogar se vuelve un módulo básico a través del cual se pueden ensamblar "comunidades" funcionales para los programas de transferencias de renta. También son comunidades funcionales para las redes clientelistas locales y fuertemente divisivas para las formas de poder tradicional basadas en la familia extendida.

El punto central es que el formato, el artefacto de la inclusión, venía con la idea explícita de "la familia como unidad de intervención" y "núcleo básico de la sociedad y la construcción de la nacionalidad" (Agencia Presidencial para la Acción Social y la Cooperación Internacional, 2010: 89). En el contexto de parentelas matrilineales que se distribuyen en el territorio, un censo por casas efectivamente implicaba intervenir la intimidad cultural (Herzfeld 2005) de los hogares y fraccionar "la familia". En otras palabras el artefacto modeló unidades sociales aptas para la política pública que trataba a los indígenas como "sujetos

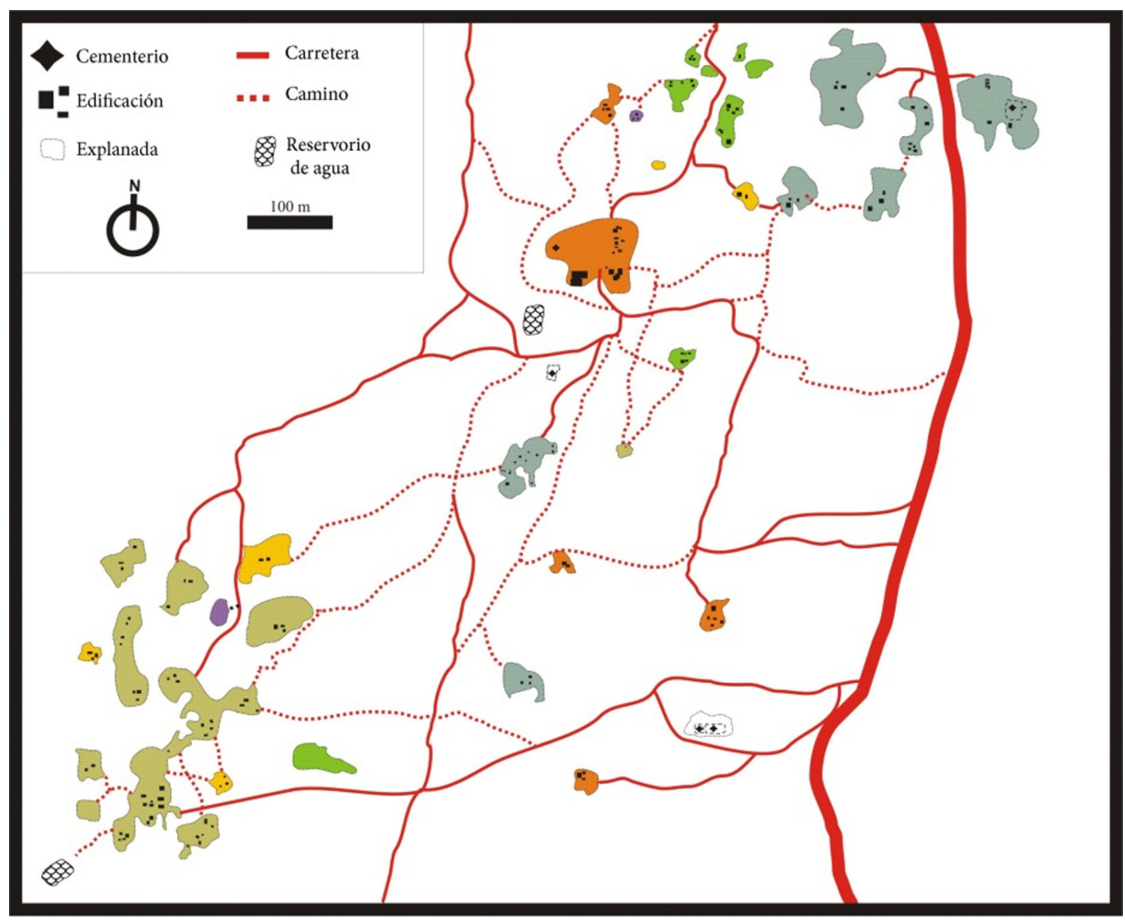

Figura 2 - Mapa de 'hogares' censados por diferentes líderes en las vecindades de la ranchería Campamento

Fuente: Tomado de Jaramillo (2014: 180) 


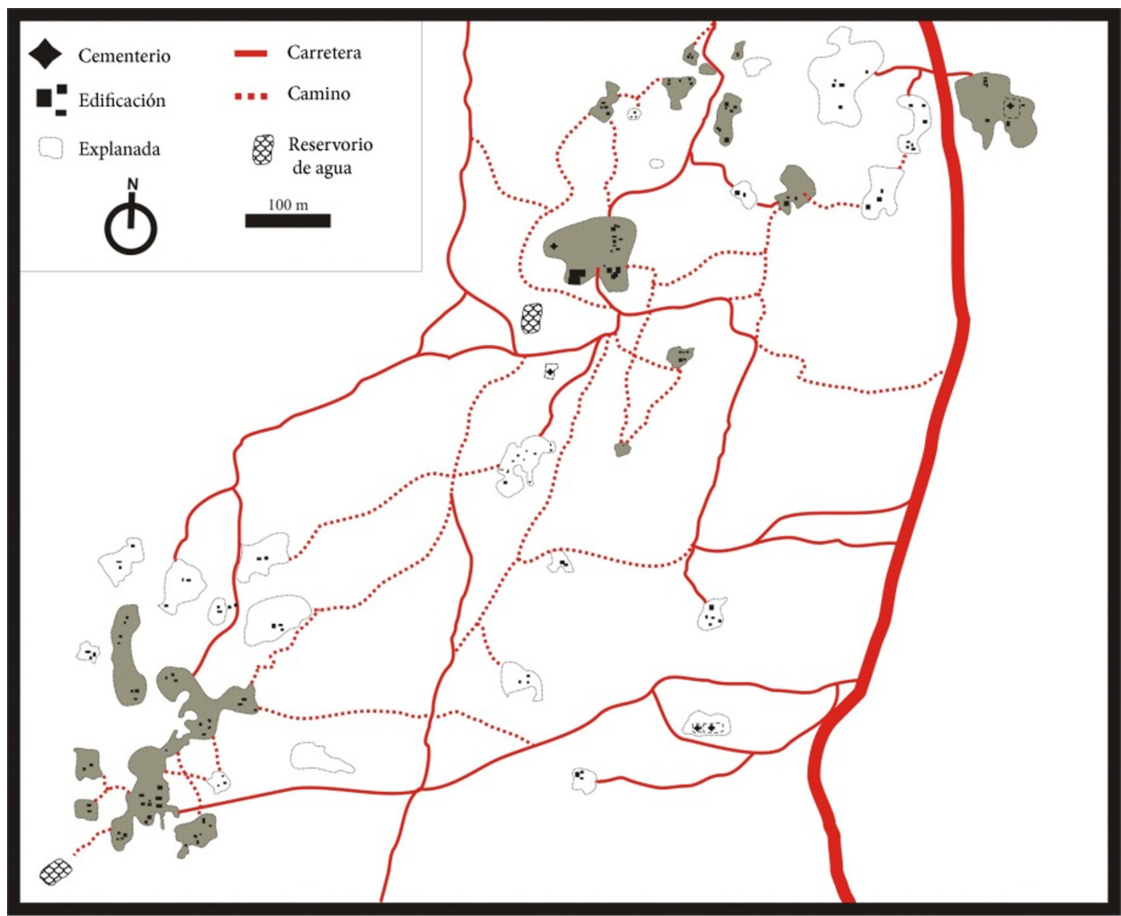

Proyectos ACH y FA (cuatro hogares en Maicao no son visibles).

Figura 3 - Mapa de hogares de la Ranchería Campamento resultado del censo de FA Fuente: Tomado de Jaramillo (2014: 181)

vulnerables" ${ }^{7}$. La encuesta se volvió un escenario de disputa íntima en un contexto donde conseguir seguidores (y registrar sus datos en el censo) resultaba en potenciales beneficios. Las mujeres de las rancherías tenían una sensación más ambigua frente a lo que estaba pasando: por un lado, la mayoría se alegraba del dinero extra que el subsidio implicaba pero, por el otro, se quejaban de las diligencias que el programa implicaba. Tampoco dejaban de recalcar que el programa estaba llevando la traición de sus familiares y a sospechas constantes de, por ejemplo, líderes que se "robaban a la gente" (refiriéndose a los datos de las personas que servían para canalizar más recursos).

Pero más allá de las implicaciones específicas lo que empezó a mostrar Familias en Acción fueron síntomas de una tendencia que después se haría patente: la creciente tecnocratización de la política social en su dimensión cotidiana. Centeno

\footnotetext{
${ }^{7}$ Los pueblos indígenas y sus derechos son centrales para la imaginación nacional desde al menos 1991, cuando la última Constitución Nacional declaró que "el Estado reconoce y protege la diversidad étnica y cultural de la Nación colombiana” (Colombia, 1992). A pesar de representar menos del 5\% de la población colombiana, el conjunto de leyes específicas para las poblaciones indígenas (que aplican en algunos contextos para afrocolombianos) fue, de acuerdo con Van Cott (2000) un símbolo de la modernización y transformación del Estado colombiano que inició en los años 80 .
} 
(1997) define la tecnocracia como el gobierno del diseño, por oposición al gobierno a través de la relación directa entre funcionarios y beneficiarios que se encuentra en la raíz de lo burocrático. El gobierno del diseño está dado inicialmente por los conocimientos expertos que permiten la clasificación y administración de los beneficiarios. Pero en su escala más cotidiana, la tecnocratización es posible gracias a la proliferación de nuevos artefactos y la instrumentalización de los ya existentes para desplegar la clase de relaciones y sujetos requeridos por el programa mismo. Esto se haría mucho más evidente con la introducción de Más Familias en Acción.

Mujeres como Dolores Pushaina, cuyas rancherías fueron disgregadas por las políticas divisivas y ahora lo que era una tiene tres nombres distintos, rápidamente adoptaron el lenguaje de Familias en Acción y dedicaron buena parte de sus semanas a hacer seguimiento en la radio local a las últimas. Las reuniones, para las cuales debía desplazarse más de dos horas desde su casa se volvieron frecuentes y ella sincronizó sus semanas, en gran parte, alrededor de las citas que le implicaba el programa. El subsidio, no obstante, llega sólo esporádicamente y cuando lo hace le representa más de un día de diligencias para reclamarlo. En nuestras conversaciones (telefónicas y presenciales), que se extienden hasta el día de hoy, un tema obligado es sobre "llenar papeles", aplicar a servicios del estado a través formularios que se multiplican, en espera de algo nuevo.

\section{Más Familias en Acción (2010-Presente)}

Maria Elsa es una mujer afrodescendiente nacida en el municipio de Barbacoas, Nariño. En Barbacoas, un municipio que ha sufrido cruentamente el conflicto armado por el enfrentamiento entre guerrillas y paramilitares (y el Ejército colombiano), ella fue parte de la primera generación de beneficiarias de Familias en Acción ${ }^{8}$. También allí recibió su primera tarjeta bancaria que finalmente quedó obsoleta una vez tuvo que verse obligada a desplazarse a causa de la persecución de hombres armados a su familia. Viajó primero a Cali, donde tiene parte de su familia, y luego a Bogotá. Allí la conocí por primera vez en el 2012, haciendo fila para la entrega de la ayuda humanitaria. Se estableció cerca de su familia en el barrio Danubio Azul. También estuve cuando intentó registrarse de nuevo en Familias en Acción, esta vez a través de un call centre que le indicaba el lugar de su cita con un SMS en el celular. Dos años más tarde, en Julio del 2014, Maria Elsa estaba pendiente aún de recibir el primer desembolso de Familias en Acción y estaba a punto de recibir el segundo desembolso de ayuda humanitaria. Mientras tanto, su barrio se había convertido en una Zona Libre de Pobreza Extrema (ZOLIP), territorios donde se ha intensificado la acción de instituciones del estado (ver la siguiente sección) y, como en otros lugares, se han establecido estrategias de "innovación

\footnotetext{
${ }^{8}$ Los derechos multiculturales para las poblaciones afrodescendientes son mucho más específicos y limitados que para los pueblos indígenas. Se circunscriben a las poblaciones rurales de las costas del país, por oposición a las grandes cantidades de habitantes urbanos, donde es más común una identidad racializada y que da cuenta del amplio uso de la categoría "negro" o "moreno".
} 
social". Maria Elsa ha sido, pues, progresivamente "incluida”, pero este proceso ha consistido en la simultánea marginación violenta y el aprendizaje de los medios adecuados para acceder a la asistencia. A la vez, recibir determinadas formas de asistencia no es independiente de cómo sus lugares de vida han sido, a la vez, marginados social, económica e, incluso, emocialmente.

En agosto de 2010, el presidente Juan Manuel Santos tomó posesión de su cargo. Heredero de su antecesor (Álvaro Uribe Vélez 2002-2006/2006-2010, Partido Colombia y luego Partido de la U), pero ansioso por diferenciarse de él, llevó a cabo un rediseño del programa que ahora se llamaría Más Familias en Acción. Acción Social pasó así a llamarse el Departamento para la Prosperidad. Los montos de las transferencias (para el 2012) habían aumentado substantivamente (entre 27,9 y 55,8 para nutrición y 16,7 y 66,9 USD para educación. La cobertura también aumento en cien mil familias y alcanzó 2,6 millones (Comissão Económica para a América Latina e Caraíbas, 2014). Sin embargo, el hito fundamental en este contexto fue la declaración del programa como Ley 1.532 (Colombia, 2012) y política permanente de estado. Esta fue mi primera experiencia con el programa en zonas urbanas del país ${ }^{9}$. Los cambios más importantes de MFA consistieron en el despliegue de una verdadera obsesión tecnológica a la que mujeres desplazadas provenientes de zonas rurales, como Maria Elsa, experimentaron con ansiedad.

Sería Rosa Bogotá, sin embargo, una activa líder que había sido Madre Líder (mujeres seleccionadas como enlace del programa con la población para temas de formación y registro) por varios años, quien primero me ayudaría a notar las contradicciones a las que estaba llevando este despliegue. Las Madres Líderes fueron una figura de intermediación entre programa y beneficiarios que operó como forma de promover la vinculación de nuevas beneficiarias en sus años de expansión más agresiva. Las Madres Líderes también era quienes convocaban y a veces moderaban los "encuentros de cuidado", escenarios de formación en los nuevos (y viejos) valores que estaba promocionando el programa: lactancia sana, buena alimentación, crianza adecuada, entre otros. Durante estos años Rosa realizó numerosas "capacitaciones": informática, contabilidad, administración de la economía doméstica. Todos los certificados sobre estos procesos de formación cuelgan de la pared del espacio de su casa donde recibía visitas y realiza reuniones con otros líderes del barrio. En un lugar más resguardado de su casa, Rosa tenía un computador de escritorio que había adquirido de segunda mano. El computador era un trofeo para ella: estaba orgullosa que sus hijos tuvieran un lugar para hacer tareas, pero sobre todo, había cambiado completamente su manera de trabajar. Antes Rosa repartía el correo. Ahora, tenía la capacidad de subcontratar repartidores del correo cuyas entregas controlaba a través de una hoja de cálculo en Excel que ella misma había diseñado: al hacerse competente tecnológicamente,

\footnotetext{
${ }^{9}$ El proyecto (codirigido con Decsi Arévalo y Oscar Rodríguez) “Efectos de las políticas sociales de subsidios en la cotidianidad de las mujeres beneficiarias. Las experiencias del Régimen subsidiado en salud y Familias en Acción" (Financiado por La Universidad de los Andes) fue parte de LATINASSIST. Además del componente cuantitativo, seguimos 10 casos de madres beneficiarios durante 10 meses.
} 
Rosa también había creado una forma de ganar buen dinero a costa de sus vecinos. Rosa también tiene una cuenta bancaria del programa, maneja hábilmente el teléfono celular. Era una madre ejemplar, según un funcionario del programa MFA que nos la presento.

Por otro lado, sin embargo, los escenarios de relacionamiento directo entre beneficiarios y el programa se redujeron a un mínimo durante estos años de reforma administrativa. Esto implicó que el cargo de Madre Líder se acabó. Se habían convertido en el chivo expiatorio de las prácticas clientelistas en el programa, pues por su contacto con otras mujeres podían conseguir votos en época electoral (Camargo; Rodríguez-Raga, 2011). Rosa había sido efectivamente alguien que había sabido convertir su intermediación con las madres en potencial político. Su cercanía con los funcionarios de Familias en Acción también le daba una vía directa a la información y, de hecho, a servicios como los talleres de formación en sistemas a través de los cuales se había vuelto tan avezada en el manejo de hojas de cálculo.

A cambio de las madres líderes el programa promovió múltiples medios tecnológicos para que los futuros y presentes beneficiarios se relacionaran con el programa. El primer medio consistió en la puesta a disposición de los usuarios de las bases de datos que determinaba su elegibilidad para el programa. A través de la página de internet del SISBEN (Sistema de Identificación de Potenciales Beneficiarios de Programas Sociales, 2014) Las mujeres (y, dependiente del programa, hombres también) podrían revisar su propia elegibilidad para diferentes programas de asistencia (Figura 4). Ellos tenían la capacidad de revisar si serían potenciales beneficiarios en las inscripciones al programa. En términos de asistencia, esta movida hacia el acceso a las bases de datos tiene implicaciones que no pueden pasar desapercibidas: los pobres deben hacer su propia selección y son ellos

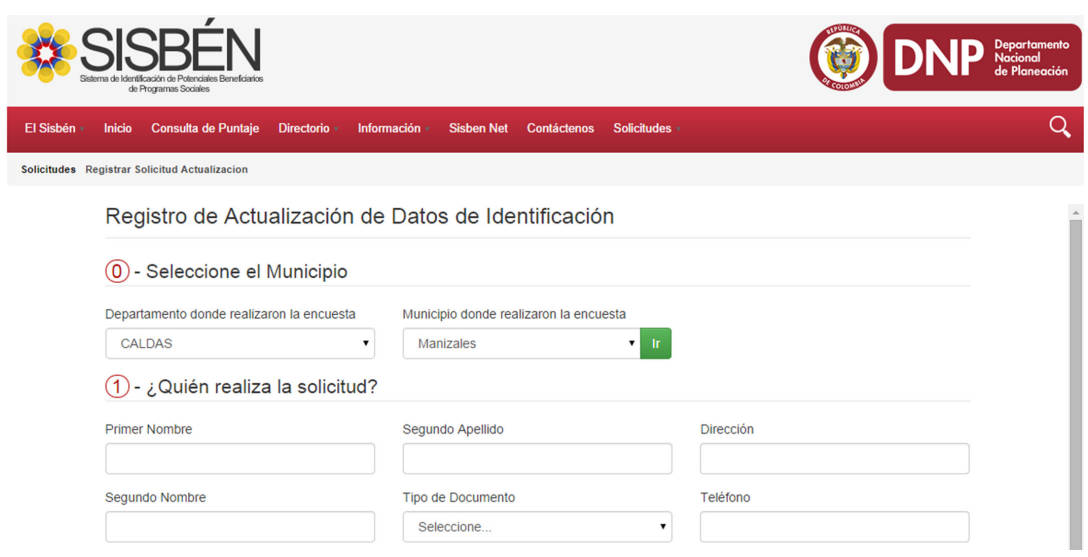

Figura 4 - Aplicación en línea para que beneficiarios consulten los programas sociales a los que pueden aplicar

Fuente: Sistema de Identificación de Potenciales Beneficiarios de Programas Sociales (2014) 
mismos quienes hacen todo el trabajo sucio en la administración del cuidado. Con esto además se materializa una ciudadanía modular y discrecional identificada en Brazíl y Chile (Georges; Ceballos, 2014; Georges, 2014) La democratización del acceso a los indicadores sociales, más que disminuir la especulación sobre el funcionamiento del programa, la remplazó por la sospecha entre vecinos. Las bases de datos eran citadas en reuniones donde las personas contrastaban entre sí sus propias representaciones sobre la pobreza con los indicadores. Paradójicamente, la alienación de las personas no condujo a la crítica de los indicadores sino a la demanda pública por su revisión. No es muy sorprendente que las protestas públicas fueran respondidas creando una página web donde las personas pueden solicitar la actualización de sus datos (aunque no de su puntaje) (Sistema de Identificación de Potenciales Beneficiarios de Programas Socialesm, 2014).

Otra innovación fundamental fue el uso de call centers y SMS en el celular para programar citas de inscripción. Las mujeres con las que realizamos el trabajo de campo durante el 2012 se quejaban recurrentemente de la dificultad en el sistema. Muchas, sin embargo, terminaron usándolo porque ahorraba tiempo. A Maria Elsa, como a otras mujeres desplazadas, les gustaba el sistema porque les evitaba enfrentarse a la ciudad desconocida y a la discriminación en los espacios burocráticos, pero todas pidieron ayuda en las inscripciones a sus hijos y conocidos (como nosotros). Maria Elsa se lo solicitó al pastor de la iglesia evangélica (Pentecostal) de la que es feligresa.

El último medio tecnológico usado en esta ola de innovación tecnológica para la asistencia tuvo como objeto la intensificación de la bancarización. A esta se le ha venido a llamar "inclusión financiera” y se creó un órgano público-privado que la promueve (Banca de las Oportunidades). Esta inclusión se ha reflejado en la variedad de medios de entrega de los “incentivos" (dinero): en Más Familias en Acción estos medios incluyen "Cuentas de Ahorro Electrónico de Trámite Simplificado" con la que se entrega una tarjeta débito; los llamados "monederos electrónicos" que usan tarjetas monederos y banca móvil (teléfonos celulares) (Departamento para la Prosperidad Social, 2014). Estas son las opciones más usadas pues los giros directos y la entrega en oficinas se reserva para muy pocos casos (indígenas y desplazados principalmente). Las mujeres beneficiarias reciben una tarjeta plástica o, en el caso de banca móvil, se asocia su cédula (que recibe el desembolso) con su número de teléfono celular para que ellas puedan obtener un código en pantalla a través de un SMS. Con el código, las mujeres pueden dirigirse a un cajero electrónico a retirar el dinero. Un efecto que noté durante el trabajo de campo fue que estas estrategias facilitan la creación de cuentas domésticas, la separación y marcación (cf. Zelizer, 2011) de dineros del hogar. El elemento celebrado de esto es que permite independencia financiera a las mujeres. Pero la creación de cuentas también implica la sospecha en el hogar y las acusaciones íntimas de robo entre sus miembros.

En conjunto, MFA ha operado a través de una intensificación de técnicas para gobernar la intimidad con artefactos que ya estaban en la vida de las personas de 
alguna manera. Los artefactos de la asistencia de MFA son artefactos de la vida cotidiana que han sido reutilizados. Lo que aspiran es a lograr un borramiento de la distinción entre vida cotidiana y vida burocrática; entre labor doméstica y pública, pues el buen uso de los artefactos está atravesado por lenguajes morales de la honestidad (en el caso de las bases de datos) y la responsabilidad (en el caso de la entrega de registros). Más allá de este borramiento, la tendencia descrita también se enmarca en una tendencia de la optimización de costos, masificación de la escala y responsabilización del beneficiario en la política social. En efecto, como mostraré en la última sección los usos potenciales son mucho más difíciles de anticipar.

\section{Red Unidos/ZOLIP}

El Danubio Azul, lugar privilegiado de nuestro trabajo de campo y hogar de Maria Elsa, se convirtió en una Zona Libre de Pobreza Extrema (ZOLIP) en el año 2013. Estas Zonas son territorios donde se da atención prioritaria a la población y seguimiento hogares en el cumplimiento de "metas". En concreto, esto implicaba que un programa de asistencia llamado Red Juntos que había surgido paralelamente a la expansión de FA a las grandes ciudades (el año 2006) y conocido como Red Unidos desde el 2010, sería intensificado. El programa es administrado por la Agencia Nacional para la Superación de la Pobreza Extrema (ANSPE), una entidad adscrita al Departamento para la Prosperidad Social (DPS) (que administra a MFA). Como el programa PAIF en Sao Paulo y Puente en Chile, la Red Unidos busca, específicamente, reducir la pobreza extrema a través de la inclusión de familias seleccionadas en la base de datos del SISBEN ${ }^{10}$. A través de un seguimiento personalizado de "cogestoras" (mujeres generalmente pobres y de los mismos barrios) a los hogares, las familias deben llegar a superar 45 "logros", divididos en 9 dimensiones, los cuales son supervisados en una base de datos en un computador portátil del programa (Agencia Nacional para la Superación de la Pobreza Extrema, 2014). Las dimensiones son identificación, ingresos y trabajo, educación y capacitación, salud, nutrición, habitabilidad, dinámica familiar, bancarización y ahorro, y acceso a la justicia. Entre los logros están, por ejemplo,

3. La familia incluida en el SISBEN tiene registrada la información personal de cada uno de sus miembros, exactamente igual a como aparecen los documentos de identidad vigentes a sus rangos de edad. (D1)

11. Las personas que lo deseen, una vez concluido el ciclo básico, se vinculan a la educación media, técnica, tecnológica o acceden a programas de formación para el trabajo. (D3)

20. Las personas con discapacidad tienen acceso a los programas de rehabilitación (en particular aquellos basados en la comunidad) y a las ayudas técnicas necesarias para su autonomía. (D4)

\footnotetext{
${ }^{10}$ El programa es similar a PDZP en México, Puente en Chile
} 
34. La familia elabora y hace seguimiento a su Plan Familiar. (D7)

37. La familia con menores de 6 años conoce y aplica pautas de crianza humanizada. (D7)

40. La familia ahorra a través del sistema financiero o de mecanismos no formales. (D8)

41. La familia conoce las características de al menos uno de los siguientes servicios financieros: ahorro, crédito y seguros. (D8)

42. Las familias que lo requieren han obtenido un crédito a través de un mecanismo financiero o formal o a través de grupos de ahorro y crédito (Agencia Nacional para la Superación de la Pobreza Extrema, 2014).

Estos logros dan a ver que la Red Unidos implica también una mezcla de acceso a capacidades (mucha de ellas técnicas) y gobierno de la intimidad. Entre las primeras se privilegian aquellas que integran a la familia al mercado. Entre las segundas las que dan evidencia de "corresponsabilidad". Las ZOLIP vienen acompañadas, por ejemplo, de ferias de servicios estatales y no estales. En el Danubio Azul, en febrero del 2013, se realizó una de estas ferias. Seis de las trece entidades participantes apuntaban a la inclusión financiera (es decir, eran bancos o instituciones de crédito).

En su conjunto, ZOLIP es la frontera última de la asistencia, abarca los casos difíciles y, en cuanto tal, han sido demarcados territorialmente. Aunque la implementación de Unidos se da a escala nacional, la distribución geográfica de las ZOLIP es diagnóstica del mismo movimiento que FA tuvo en sus orígenes al concentrarse en territorios problemáticos, extremadamente vulnerables. Sin embargo, a diferencia del programa TCE, ZOLIP también se ha insertado en las áreas más marginales de las principales capitales del país: metafóricamente se concentran en la intimidad del hogar simbólico del proyecto nacional (Bogotá).

Guzmán (2013) ha observado detalladamente como operan las relaciones entre beneficiarias y cogestoras en el caso de la ZOLIP de un municipio vecino de Bogotá. El programa se ha promocionado a través del discurso de la corresponsabilidad y empoderamiento, pero esto realmente puede existir a través de la intrusión en la vida privada. Las visitas de las cogestoras ha sido descrita por Guzmán (2013) como una disputa por el control del tiempo y el espacio del hogar. La manifestación más básica de esta disputa entre beneficiarias es "hacer perder el tiempo"; es decir, el incumplimiento de citas, hacer esperar, dilatar las visitas, que son acciones emprendidas tanto por beneficiarias como por cogestoras. Prácticas más elaboradas consisten en dar regalos, que implican maneras de imbuir la relación burocrática de compromisos íntimos futuros.

Dicho lo anterior, es vital subrayar que es la base de datos que lleva la cogestora en su computador portátil la que realmente gobierna la relación. En esto el programa es el que se asegura el control no solo de los tiempos íntimos y cotidianos, sino del 
futuro mismo. El acompañamiento a las familias se hace a través de la base de datos, un software llamado "Verificación de Logros", que se alimenta in situ y que se sincroniza al sistema de información nacional en tiempo real. El software tiene tantas casillas como logros que se reconocen a través de colores (Figura 5). La cogestora, va registrando el progreso en sus visitas y ella misma va incluyendo la evidencia que llevará a que la familia sea excluida de la categoría de "pobre extrema". Pero la base de datos gobierna más que el tiempo íntimo de la familia porque con base en estas se toman las decisiones en los comités técnicos. En uno de estos a los que tuve la oportunidad de asistir, cada logro era revisado de acuerdo al número de familias que no lo cumplen. Inmediatamente se delega un representante de un ente estatal para ultrafocalizar estos casos, cuyos detalles pueden ser consultados, en tiempo real, durante la reunión.

Por último, la estrategia ZOLIP está articulada a un componente de la ANSPE llamada Centros de Innovación Social. En el marco de esta estrategia se hacen proyectos puntuales de acceso a recursos tecnológicos y de emprendimiento. En las vecindades de Bogotá, por ejemplo, este ha sido la entrada a One Laptop per Child (OLPC), una ONG norteamericana apoyada por Intel que promueve justamente lo que su nombre dice y se apoya en grandes mentes del emprendimiento global. En otros municipios se han creado proyectos de emprendimiento, de "negocios sociales" y la experimentación con tecnología para el mejoramieno de vivienda y la sanidad", según el Centro de Innovación Social de la ANSPE (Agencia Nacional para la Superación de la Pobreza Extrema, 2014).

$\star \star \star *$

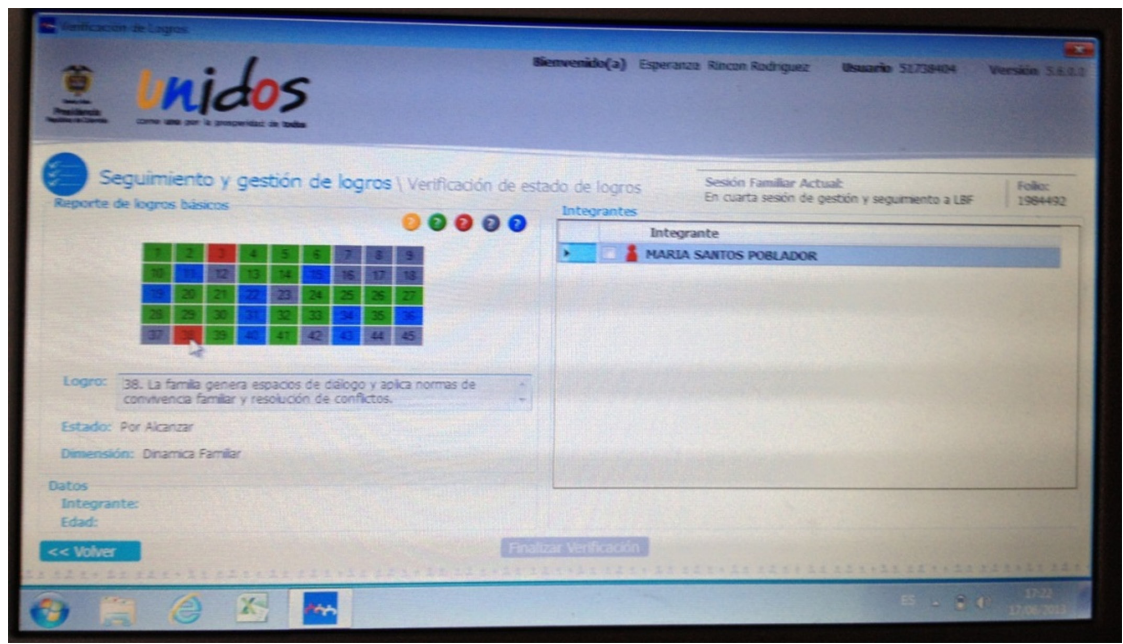

Figura 5 - Interfaz del programa en el computador de una cogestora.

Fuente: Tomada por Yenny Guzmán (2013) 
En resumen, las tres estrategias implican dimensiones distintas, pero complementarias, de una forma de gubernamentalidad futurista centrada en la intimidad del hogar y la vida cotidiana. Esta forma de gubernamentalidad espera que la tecnología, por sí misma cierre la brecha entre centro y periferia, cree la relación social y "engrane" a los ciudadanos en las publicitadas estrategias de "encadenamiento", "articulación" y "sinergias": metáforas que asumen a los estados y a los ciudadanos como componentes tecnológicos.

\section{Del hechizo tecnológico al "hechizo" tecnológico}

Las relaciones con la tecnología que he presentado en este artículo apuntan primariamente a gobernar la intimidad. Aunque es necesario investigar más sobre el uso cotidiano de la tecnología por parte de los y las beneficiarias, es obvio que lo que hacen estas últimas con los artefactos de la asistencia supera la imaginación del programa. . Lo que me lleva finalmente a dos formas de relación de las personas con la tecnología y que, de hecho, entran en tensión en la configuración del hogar como unidad de intervención.

En mayo de 2014, una de las mujeres que hizo parte del proyecto durante el 2012 me contactó a través de Facebook. Estaba arrestada en su casa. Ella nunca había ocultado algunos vínculos con el mundo delincuencia del barrio. Según ella misma, un computador portátil que tenía durante nuestro trabajo de campo, así como su celular, eran robados. Ella había entrado a ser directamente parte de una banda y había sido arrestada. Por su condición de madre de familia había recibido el beneficio de casa por cárcel. El deseo por los artefactos tecnológicos es la contraparte del optimismo tecnológico con el que opera la política social. Hay un patente círculo vicioso aquí: la mujer pobre llega a ser criminal por aspirar demasiado lo que aprendió a desear.

Alfred Gell (1992) dijo que la tecnología involucra a las personas en redes de intencionalidad en las cuales se distribuye la agencia. A esto le llamo el encantamiento de la tecnología y es, justamente lo que parece ir implícito en la inclusión de tecnologías y artefactos en la asistencia. No obstante esta suerte de encantamiento de la tecnología también opera, literalmente, como un hechizo que margina, vuelve ansiosas a las personas sobre sus propias aspiraciones y finalmente las encierra en guetos de ignorancia, inhabilidad, criminalidad y la acumulación de basura tecnológica en las casas. Por supuesto, también aparatos como los teléfonos celulares han llegado a ser instrumentos clave en la configuración de redes de cuidado y empleo entre las mujeres, pero esto también se encuentra de la imagen específica que el programa tiene del hogar como autocontenido y autosuficiente.

Otra forma de "hechizo" más interesante es el que se puede encontrar en muchos apartes de este artículo. En su reflexión sobre la innovación tecnológica en países del sur global, Hernán Thomas (1995) ha manifestado que los modelos existentes son, en realidad, aquellos del norte. En países como Argentina, Brasil y Colombia, la innovación toma la forma de la contra-ingeniería, de desarmar y 
rearmar que él llama reciclaje tecnológico. En Colombia esto toma el nombre de "hechizos", cosas hechas con medios limitados y materiales disponibles, fragmentos de segunda mano, entre otras cosas. Los hechizos aparecen en el computador de Rosa, en la manera como mucha gente hace operar su teléfono celular a través de cargadores genéricos de baterías y piezas falsificadas que provienen del mundo de la globalización no-hegemónica. Entender estos componentes de la innovación de los hogares puede llevar a lugares revolucionarios que aún están por explotar completamente. También están en el centro de formas de sociabilidad emergente entre las personas sujeto de las políticas de la asistencia.

\section{Conclusiones}

Este artículo ha sido una reflexión sobre el gobierno tecnocrático y el territorio en Colombia. Pero también en regímenes de gobierno neoliberal. También es una reflexión sobre mi trayectoria como investigador de la asistencia social y la manera como he notado la centralidad de la tecnología en la asistencia. A menudo se piensa que la tecnocratización implica universalización, pero la política social conserva una geografía propia del estado liberal. Esta diferencia corresponde a la distinción paradigmática entre centro y periferia. La diferencia no es necesariamente geográfica, sino en sus propios términos, una diferencia establecida a través de la capacidad técnica de manipular el mundo.

En este artículo he ofrecido una caracterización de una forma de gubernamentalidad que aspira a que la tecnología y los artefactos logren la incorporación de "los hogares" al proyecto económico, político y moral de un país evidentemente inclinado al proyecto neoliberal. El problema del estado neoliberal no es gobernar solamente gobernar a través del imperativo de la libertad, sino dar a las personas los medios y cosas a través de las cuales su libertad puede ser concretada (Bennett, 2009). Pero el problema va más allá de los medios y los fines: en el diseño de las cosas también va implícito parte del gobierno de las relaciones más íntimas. Esto se refleja en la asistencia. El estado está obsesionado con la tecnología como forma de justicia. Sin embargo lo que yo documento es la simultánea capacidad gobernar a través de la tecnología ha ido en paralelo (y no necesariamente que se causen mutuamente) a una re-espacialización de la desigualdad.

\section{Referencias}

AGENCIA NACIONAL PARA LA SUPERACIÓN DE LA POBREZA EXTREMA - ANSPE.

Bogotá. Disponible: <http://www.anspe.gov.co/es/programa/estrategia-unidos/ dimensiones-y-logros>. Acceso: 10 sept. 2014.

. Centro de Innovación Social. Bogotá. Disponible: $<$ http://issuu.com/ciscolombia/ docs/cis_2013>. Acceso: 10 sept. 2014. 
AGENCIA PRESIDENCIAL PARA LA ACCIÓN SOCIAL Y LA COOPERACIÓN INTERNACIONAL, Departamento Nacional de Planeación - DPN. El camino recorrido diez años de Familias en Acción. Bogotá, 2010.

BASIAGO, Andrew. The limits of technological optimism. Environmentalist. Hampshire, v. 14, n. 1, 1994, pp. 17-22. http://dx.doi.org710.10077bf01902656.

BENNETT, Tony. Liberal government and its outsides: introductory notes. In: $4^{\circ}$ Government and Freedom Seminar. Milton Keynes: The Open University, pp. 1-7, 2009.

BRICKELL, Katherine. Geopolitics of home. Geography Compass, Hoboken, v. 6, n. 10, 2012, pp. 575-588. http://dx.doi.org/10.1111/j.1749-8198.2012.00511.x.

CAMARGO, Gabriel Nicolás, RODRÍGUEZ-RAGA, Juan Carlos. Familias en acción y preferencias políticas, Colombia 2010. Apuntes de Cultura Política: desde el Observatorio de la Democracia, Bogotá, n. 1, 2011, pp. 1-8.

CENTENO, Miguel Ángel. Redefiniendo la tecnocracia. Desarrollo Económico, Buenos Aires, v. 37, n. 146, 1997, pp. 215-240.

COLOMBIA. República de Colombia. Constitución política de Colombia 1991. Bogotá, Departamento Administrativo del Servicio Civil, 1992.

. Ley $n^{\circ} 1532$. Por medio de la cualse adoptan unasmedidas de política y se regula el funcionamiento del programa Familias en Acción. Bogotá, Congreso de Colombia, 2012.

. Governo. Presidencia de la República. Las ZOLIP en Bogotá. Bogotá. Disponible: $<$ http://wsp.presidencia.gov.co/bogota/prensa/2013/Zolip/Paginas/Las-ZOLIP-enBogota.aspx>. Acceso: 7 sept. 2014.

COMISSÃO ECONÓMICA PARA A AMÉRICA LATINA E CARAÍBAS - CEPAL. Programa de Transferencias Condicionadas. Más familias en acción (2001-). Santiago, 2014. Disponible: $<$ http://dds.cepal.org/bdptc/programa/componentes.php?id=12>. Acceso: 10 sept. 2014.

DEPARTAMENTO PARA LA PROSPERIDAD SOCIAL - DPS. Más familias en acción: más compromiso por la equidad. Bogotá, 2014. Documento Operativo Técnico DOT, n. 13. Entrega de Incentivos.

FISZBEIN, Ariel et al. Conditional cash transfers: reducing present and future poverty. Washington, World Bank, 2009.

FOUCAULT, Michel. Governmentality. In: BURCHELL, Graham; GORDON, Collin; MILLER, Peter (Eds.). The Foucault effect: studies in governmentality. London, Harverster Wheatsheaf, 1991.

GELL, Alfred. The technology of enchantment and the enchantment of technology. In: COOTE, Jeremy; SHELTON, Anthony (Eds.). Anthropology, art and aesthetics. Oxford, Oxford University Press, 1992, pp. 40-63.

GEORGES, Isabel; CEBALLOS, Marco. Bolsa familia y la asistencia social en Brasil: de la lucha política a la mercantilización local. Caderno CRH, Salvador, v. 27, n. 72, 2014, pp. 513-529. http://dx.doi.org/10.1590/S0103-49792014000300005. 
GEORGES, Isabel. Reconfiguration des politiques sociales au Brésil. Paris, La Découverte, 2014. http://dx.doi.org/10.3917/tgs.032.0045.

GUZMÁN, Yenny. Burocracia a domicilio: una mirada etnográfica a la política social contra la pobreza extrema en Chía. Bogotá, Uniandes, 2013. Tesis (Antropólogo).

HANDELMAN, Don. Bureaucratic transactions: the development of official-client relationships in Israel. In: Kapferer, Bruce (Ed.). Transaction and meaning: directions in the anthropology of exchange and symbolic behavior. Philadelphia, Institute for the Study of Human Issues, 1976, pp. 223-275.

HERZFELD, Michael. The social production of indifference: exploring the symbolic roots of western bureaucracy. Chicago, University of Chicago Press, 1993. Disponible: $<$ http:// www.loc.gov/catdir/description/uchi052/93001674.html>. Acceso: 20 enero 2016.

Cultural intimacy: social poetics in the Nation-State. 2. ed. New York, Routledge, 2005. Disponible: <http://www.loc.gov/catdir/toc/ecip0417/2004008898.html>. Acceso: 20 enero 2016.

JARAMILLO, Pablo. Etnicidad y victimización: genealogías de la violencia y la indigenidad en el norte de Colombia. Bogotá, Uniandes, 2014.

. La duplicidad de las cédulas: valor, crisis moral e indigenidad en la Guajira. In: FORERO, Olga Restrepo (Ed.). Ensamblando heteroglosias. Bogotá, Colciencias, Universidad Nacional, 2013, pp. 394-405.

KLEINE, Dorothea. Technologies of choice?: ICTs, development, and the capabilities approach. Cambridge, Mass, MIT Press, 2013. The Information Society Series.

LATOUR, Bruno. The pasteurization of France. Cambridge, Harvard University Press, 1988.

. Reassembling the social: an introduction to actor-network-theory. Oxford, Oxford University Press, 2005.

LAW, John. Actor network theory and material semiotics. 2007, pp. 1-21. Disponible: $<\mathrm{http}$ ://www.heterogeneities.net/publications/Law-ANTandMaterialSemiotics.pdf >. Acceso: 20 enero 2016.

MANN, Michael. The sources of social power. New York, Cambridge University Press, 1986. Disponible: <http://www.loc.gov/catdir/description/cam023/85014962.html>. Acceso: 20 enero 2016.

OSWIN, Natalie; OLUND, Eric. Governing intimacy. Environment and Planning D: Society and Space, London, v. 28, n. 1, 2010, pp. 60-67. http://dx.doi.org/10.1068/d2801ed.

RESTREPO FORERO, Olga (Ed.). Ensamblando estados. Bogotá, Universidad Nacional de Colombia, Colciencias, 2013a.

Ensamblando heteroglosias. Bogotá, Colciencias, Universidad Nacional de Colombia, 2013b.

SEN, Amartya Kumar. Development as freedom. Oxford, Oxford University Press, 2001.

SISTEMA DE IDENTIFICACIÓN DE POTENCIALES BENEFICIARIOS DE PROGRAMAS SOCIALES - SISBÉN. Bogotá, 2014. Disponible: <https://www.sisben.gov.co/ ConsultadePuntaje.aspx>. Acceso: 20 enero 2016. 
. Registro de actualización de datos de identificación. Bogotá. Disponible: $<$ https://www.sisben.gov.co/Solicitudes/RegistrarSolicitudActualizacion.aspx >. Acceso: 20 enero 2016.

THOMAS, Hernán. Surdesarrollo: producción de tecnología en países subdesarrollados. Buenos Aires, Centro Editor de América Latina, 1995.

UNITED STATES GOVERNMENT ACCOUNTABILITY OFFICE - GAO. PLAN COLOMBIA: drug reduction goals were not fully met, but security has improved; U.S. agencies need more detailed plans for reducing assistance. Washington, GAO, 2008. Report to the Honorable Joseph R. Biden, Jr., Chairman, Committee on Foreign Relations, U.S. Senate Report.

VAN COTT, Donna Lee. The friendly liquidation of the past: the politics of diversity in Latin America. Pittsburgh, University of Pittsburgh Press, 2000. Pitt Latin American Series.

WINNER, Langdon. Do artifacts have politics? Daedalus, Lisboa, v. 109, n. 1, 1980, pp. 121-136.

ZELIZER, Viviana. El significado social del dinero. Buenos Aires, Fondo de Cultura Económica, 2011.

Recebido em: 20/01/2016

Aprovado em: 05/04/2016

\section{Como citar este artigo:}

JARAMILLO, Pablo. Gubernamentalidades futuristas: gobernar el hogar en la era del optimismo tecnológico. Contemporânea - Revista de Sociologia da UFSCar. São Carlos, v. 6, n. 1, jan.-jun. 2016, pp. 75-95. 